Article

\title{
Numerical and Experimental Study on Multi-Focal Metallic Fresnel Zone Plates Designed by the Phase Selection Rule via Virtual Point Sources
}

\author{
Jinseob Kim ${ }^{1}$, Hyuntai Kim ${ }^{2}$, Gun-Yeal Lee ${ }^{3,4}$, Juhwan Kim ${ }^{1}$, Byoungho Lee ${ }^{3,4}$ and \\ Yoonchan Jeong 1,4,5,* (iD \\ 1 Laser Engineering and Applications Laboratory, Department of Electrical and Computer Engineering, \\ Seoul National University, Seoul 08826, Korea; kjs8773@snu.ac.kr (J.K.); kjh2653350@snu.ac.kr (J.K.) \\ 2 Optoelectronics Research Centre, University of Southampton, Southampton SO17 1BJ, UK; \\ H.Kim@soton.ac.uk \\ 3 Optical Engineering and Quantum Electronics Laboratory, Department of Electrical and Computer \\ Engineering, Seoul National University, Seoul 08826, Korea; dlrjsduf12@snu.ac.kr (G.-Y.L.); \\ byoungho@snu.ac.kr (B.L.) \\ 4 Inter-University of Semiconductor Research Center, Seoul National University, Seoul 08826, Korea \\ 5 Institute of Applied Physics, Seoul National University, Seoul 08826, Korea \\ * Correspondence: yoonchan@snu.ac.kr; Tel.: +82-2-880-1623
}

Received: 2 March 2018; Accepted: 13 March 2018; Published: 15 March 2018

\begin{abstract}
We propose a novel design method for multi-focal metallic Fresnel zone plates (MFZPs), which exploits the phase selection rule by putting virtual point sources (VPSs) at the desired focal points distant to the MFZP plane. The phase distribution at the MFZP plane reciprocally formed by the VPSs was quantized in a binary manner based on the phase selection rule, thereby leading to a corresponding on-off amplitude pattern for the targeted MFZP. The resultant phase distribution was dependent on the complex amplitudes of the VPSs, so that they could be determined from the perspective of both multi-focal functionality and fabrication feasibility. As a typical example, we utilized the particle swarm optimization algorithm to determine them. Based on the proposed method, we designed and numerically analyzed two types of novel MFZPs-one for a monochromatic multi-focal application and the other for a multi-chromatic mono-focal application-verifying the effectiveness and validity of the proposed method. We also fabricated them onto Au-deposited glass substrates, using electron beam evaporation and a focused ion beam milling process. We experimentally characterized them and also verified that they successfully demonstrated their feasibilities. The former produced distinct hot spots at three different focal distances of 10, 15, and $20 \mu \mathrm{m}$ for monochromatic incidence at $650 \mathrm{~nm}$, and the latter produced a single hot spot at a focal distance of $15 \mu \mathrm{m}$ for multi-chromatic incidence at 660, 532, and $473 \mathrm{~nm}$. The experimental results were also in good agreement with their corresponding numerical results. We expect that both MFZPs will have various applications, such as laser micromachining, optical trapping, biomedical sensing, confocal collimation, achromatic optics, etc.
\end{abstract}

Keywords: lenses; multi-focusing; optics at surfaces; binary optics; achromatic optics

\section{Introduction}

Fresnel zone plates (FZPs) or metallic Fresnel zone plates (MFZPs) have received considerable attention in recent years as an efficient focusing element for flat optical applications [1-5]. They can be used not only as a replacement for conventional focusing lenses, but also as optical elements with exquisite properties by implementing various novel patterns onto the metallic annular slit structure, such as multi-focal, multi-chromatic, and super-oscillatory lenses [6-10]. Among them, studies of 
MFZPs for multi-focal applications have recently received considerable attention. These studies have exploited various authentic mathematical formulae as generating functions for the metallic annular slit structure, such as Fibonacci, fractal, and cantor series [11-13]. While they may lead to unique multi-focal capabilities, it is hard to say whether they can rigorously produce multi-focal points at certain predetermined locations. Moreover, there have been very few attempts to investigate a systematic design method for multi-focal MFZPs even though such a method could have a huge impact on improvements in laser micro-machining, optical trapping, chemical sensing, biomedical sensing, confocal collimation, achromatic optics, etc. [14-21]. Thus, we think that this field merits additional research.

One of the simplest and most conventional ways to realize a multi-focal MFZP is to divide the area of a lens into multiple sections, such that each section is individually designed and constructed to produce a different focal point [22-24]. Whilst this spatial division can be carried out either azimuthally or radially, it can produce some undesirable outcomes. The former cannot avoid polarization dependence issues, because the individual section naturally results in a different orientation to the polarization axis of incident light. In contrast, the latter incurs significant degradation in the sharpness of the focal depth [25], because the effective beam width allocated to each radial section is inevitably reduced. On the other hand, a more intricate way to realize a multi-focal MFZP is to exploit a pixelated phase pattern on the substrate via dielectric material deposition [26], such that all the combined diffractions eventually form multi-focal spots at different depths. However, such a highly pixelated phase pattern generally requires a complicated fabrication process, such as plasma-enhanced chemical deposition. This aspect substantially undermines the fundamental merits of MFZPs, which include the simplicity of both its design and the fabrication procedure.

We here propose and demonstrate novel multi-focal MFZPs that can overcome the issues mentioned above as well as providing a simple but powerful systematic design method for them. We exploit the use of virtual point sources, placed at the desired focal points, to obtain an effective MFZP pattern of multi-focal functionality, replying to the phase conjugation principle. We can readily fine-tune and optimize the pattern, varying the complex amplitudes of the virtual point sources, i.e., their intensity levels and initial phase offset values so that the corresponding MFZP results in the best performance from the perspective of both multi-focal functionality and fabrication feasibility. We thus present all the details of the proposed systematic design method for multi-focal MFZPs and utilize the method to configure two types of MFZPs with novel functionality, including a monochromatic multi-focal MFZP and a multi-chromatic mono-focal MFZP. We fabricated them and verified their novel characteristics both numerically and experimentally.

\section{Design Principle for a Multi-Focal MFZP}

Conventional MFZP implementation is carried out by selectively opening or closing the individual paths of light so that they may result in constructive or destructive interference at the specified focal point, respectively. Thus, assuming that the incident light is monochromatic and has a uniform phase front at the MFZP plane, i.e., $z=0$, the radii of the individual annular slits can be determined by the following formula [27]:

$$
r_{n}=\sqrt{(n+\alpha) \lambda_{0} f_{0}+\frac{(n+\alpha)^{2} \lambda_{0}^{2}}{4}}(n=1,2, \ldots, 2 N),
$$

where $r_{n}$ denotes the inner radius of an annular slit opening for an odd integer $n$ or its outer radius for an even integer $n, N$ is the total number of annular slits, $\alpha$ is the initial phase-offset constant, $f_{0}$ is the focal length, and $\lambda_{0}$ is the wavelength of incident light. Thus, this MFZP produces a hot spot at $z=f_{0}$ via all constructive interference. This design principle can be reformulated in a reciprocal manner, because without loss of generality, the propagation of electromagnetic waves is "reciprocal" in a simple medium [28]. In other words, if one puts a virtual point source (VPS) at $z=f_{0}$, where all the light is 
initially focused down, it radiates a spherical wave towards the MFZP plane. This phase-conjugated or backward-directed spherical wave eventually produces a phase distribution at $z=0$ in relation to the initial phase offset value $\alpha$. Specifically, if this phase distribution is quantized into 0 and $\pi$ in a binary manner based on the so-called phase selection rule (PSR), subsequently making the 0 and $\pi$ regions open and closed, the resultant pattern of the annular slits is the same as specified by Equation (1) for a mono-focal MFZP. Building upon this fundamental principle of the PSR via a VPS as well as the principle of superposition, one can readily design a multi-focal MFZP if one puts multiple VPSs at different focal points to obtain the resultant phase distribution at $z=0$. In principle, the rest of the procedure is identical to the mono-focal MFZP case discussed above. We visualize the method in Figure 1.

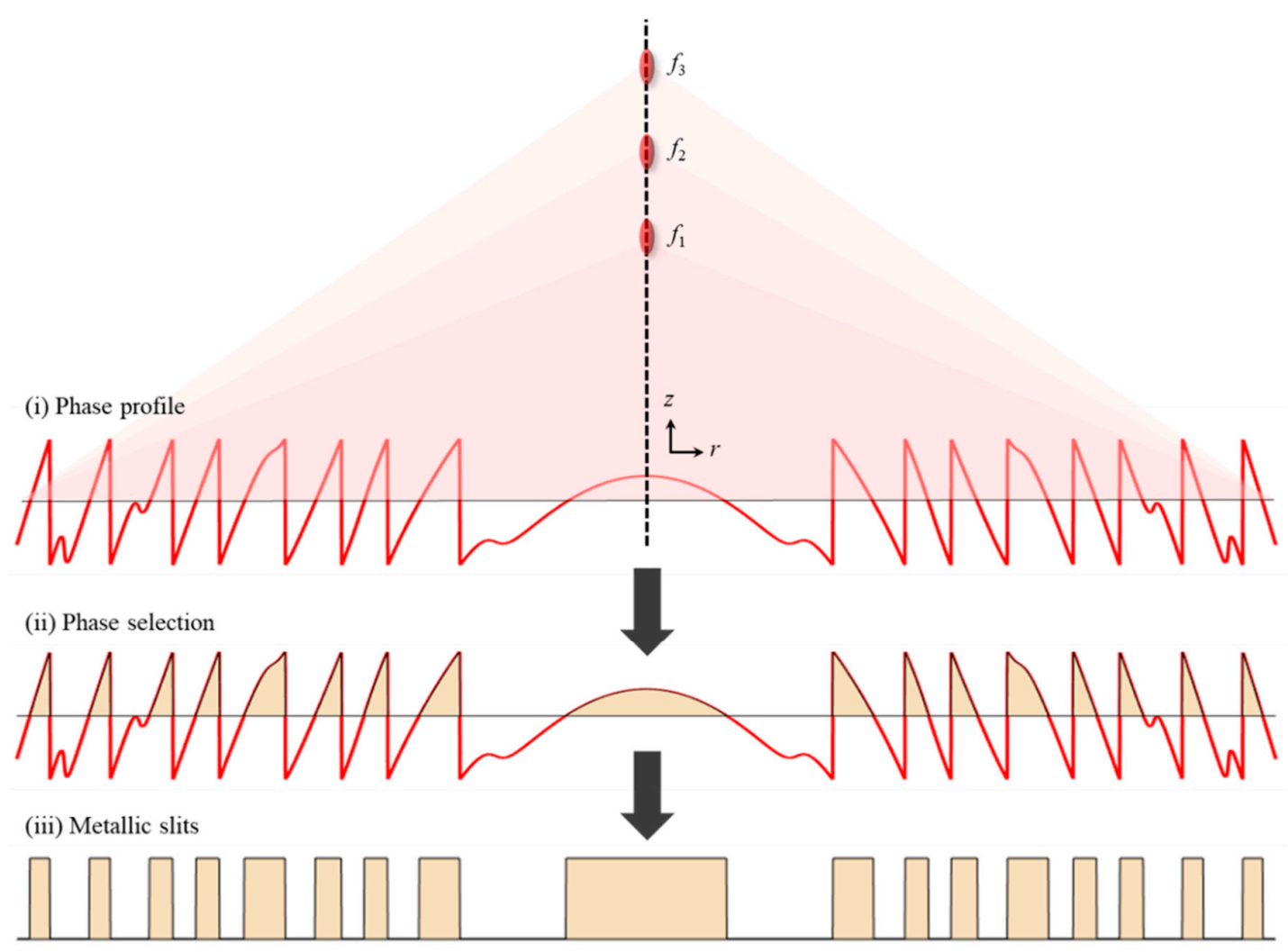

Figure 1. Design principle for a multi-focal MFZP: (i) Generation of a phase profile from the VPSs placed at different focal points. (ii) Application of the PSR, selectively taking the sections where constructive interferences take place. (iii) Creation of an on-off (open-closed) metallic slit pattern based on the binary quantization of the phase profile.

Moreover, it is worth noting that, depending on the depths of the multiple focal points, the intensities of the individual spherical waves at the MFZP plane, i.e., their influences on the resultant phase distribution, decrease based on the inverse-square law. Thus, when designing a multi-focal MFZP, one should also adjust the intensity levels of individual VPSs according to the intensity levels at the desired focal points. Consequently, considering that the quantized amplitude pattern can considerably vary in a binary manner based on the PSR, given the choice of the complex amplitudes of the VPSs, i.e., their intensity levels and initial phase-offset values, it is necessary to optimize them from the perspective of both multi-focal functionality and fabrication feasibility. In particular, we chose to use the particle swarm optimization (PSO) method [29] for this optimization procedure, although it is not necessarily the only method available. In addition, we carried out all the numerical calculations and optimizations using the commercial mathematical software Matlab (R2016b, MathWorks, Natick, MA, USA). 


\section{A Monochromatic, Multi-Focal MFZP}

To demonstrate a proof of principle for multi-focal functionality, we first designed an example MFZP capable of producing multiple hot spots at three different points with identical peak intensities for monochromatic incident light. We chose the design parameters as the following: we set the diameter of the target MFZP to $50 \mu \mathrm{m}$ and the three foci to $f_{1,2,3}=10,15$, and $20 \mu \mathrm{m}$. We assumed that the foci were on the central axis of the MFZP, i.e., the $z$-axis, and that the wavelength of the incident light was $650 \mathrm{~nm}$.

For simplicity, we initially chose the indicators of the PSO algorithm from two perspectives: (1) how accurately the three foci were formed at the desired locations in terms of $\Delta f_{m}$; and (2) how evenly the peak intensities of the three hot spots were determined in terms of $\Delta I_{m}$. However, it is worth noting that the optimization procedure can further be extended in various additional aspects if necessary. We set the penalty parameters based on those two simple criteria and carried out the optimization procedure based on the PSO algorithm until both criteria were met within the tolerance values, such that the relative tolerance values for $\Delta f_{m}$ and $\Delta I_{m}$ were $\pm 0.5 \%$ and $\pm 3 \%$, respectively. In Table 1, we summarize the target design parameters as well as the resultant parameters obtained after going through the application of the PSR via the VPSs along with the PSO algorithm. In particular, it is worth noting that we set the minimum step for the slit-width variation, i.e., the slit resolution, to $10 \mathrm{~nm}$, in consideration of the fabrication resolution of the in-house focused ion beam (FIB) system.

Table 1. Simulation parameters for a monochromatic, multi-focal MFZP.

\begin{tabular}{|c|c|c|c|c|c|c|}
\hline \multicolumn{7}{|c|}{ Design Parameters } \\
\hline \multirow{2}{*}{ Parameters } & \multirow{2}{*}{ Foci } & \multirow{2}{*}{ Wavelength } & \multirow{2}{*}{ Diameter } & \multirow{2}{*}{$\begin{array}{c}\text { Slit } \\
\text { Resolution }\end{array}$} & \multicolumn{2}{|c|}{ Tolerance } \\
\hline & & & & & Position $\left|\Delta f_{m}\right|$ & Intensity $\left|\Delta I_{m}\right|$ \\
\hline Value & $10,15,20 \mu \mathrm{m}$ & $650 \mathrm{~nm}$ & $50 \mu \mathrm{m}$ & $10 \mathrm{~nm}$ & $0.5 \%$ & $3 \%$ \\
\hline \multicolumn{7}{|c|}{ Optimized Parameters } \\
\hline Parameters & \multicolumn{2}{|c|}{ Relative Intensities $\left(I_{1}, I_{2}, I_{3}\right)$} & \multicolumn{2}{|c|}{ Initial Phase-Offset $\left(\phi_{1}, \phi_{2}, \phi_{3}\right)$} & Position $\left|\Delta f_{m}\right|$ & Intensity $\left|\Delta I_{m}\right|$ \\
\hline Value & \multicolumn{2}{|c|}{$0.13,0.36,1$} & \multicolumn{2}{|c|}{$1.05 \pi, 0.70 \pi, 2.00 \pi$} & $<0.5 \%$ & $<2.9 \%$ \\
\hline
\end{tabular}

In Figure 2, we illustrate the resultant pattern of the designed MFZP and the corresponding numerical result regarding its multi-focal functionality. It is worth noting that the intensity level at $f_{3}$ was regarded as the reference value. From the result, one can clearly see that it could produce three hot spots at the expected locations with sufficiently identical peak intensity levels. The individual locations of the three focal points were 9.95, 15.04, and $19.99 \mu \mathrm{m}$, all of which were within the specified tolerance. Considering that we simplified the optimization criteria for this MFZP for the purpose of a proof of principle demonstration, there remains room for improvement in aspects other than the location accuracy and intensity uniformity. For example, in the above simulation results, the side lobe suppression ratio was estimated to be $-6.12 \mathrm{~dB}$. One can improve this ratio significantly if one extends the optimization procedure with an additional criterion. Notwithstanding this, we emphasize that the proposed method, based on the application of the PSR via the VPSs along with the PSO algorithm, was very effective and powerful in systematically designing an MFZP with multi-focal functionality for monochromatic incident light.

Based on the designed pattern shown in Figure 2a, we fabricated a real MFZP and characterized it experimentally. The fabrication procedure was as follows: We first uniformly deposited an $\mathrm{Au}$ metal layer of $100 \mathrm{~nm}$ thickness on a glass substrate, using an electron beam evaporation method [30]. We implemented the designed pattern via the FIB milling process [18,31].

In Figure 3, we illustrate the schematic diagram of the experimental arrangement for the characterization of the fabricated MFZP. It comprised a light source, an MFZP sample, and an imaging device. We used a diode laser (VFL-250, Fibretool, Shanghai, China) operating at $650 \mathrm{~nm}$ as the light source to irradiate the MFZP sample. It is worth noting that the diode laser could be replaced by 
a red/green/blue (RGB) laser system for another experiment to be discussed in the next section. We utilized an optical microscope (BH, Olympus, Tokyo, Japan) with a complementary metal-oxide semiconductor (CMOS) camera (UCMOS05100KPA, ToupTek, Hangzhou, Zhejiang, China) as the imaging device to detect the transmitted light through the MFZP sample, varying the distance between the MFZP sample and the objective lens of the optical microscope.

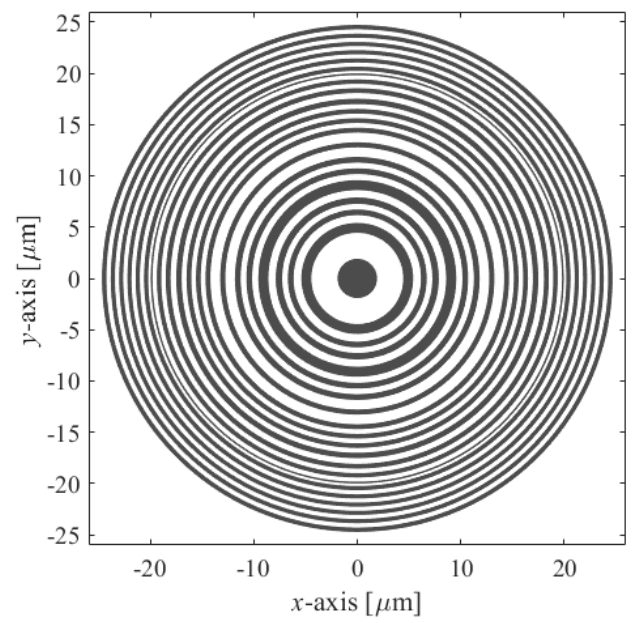

(a)

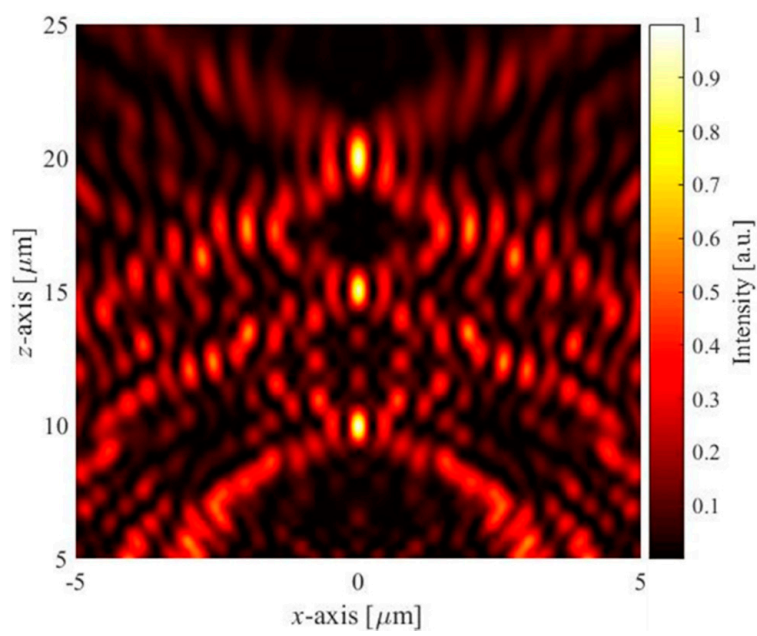

(b)

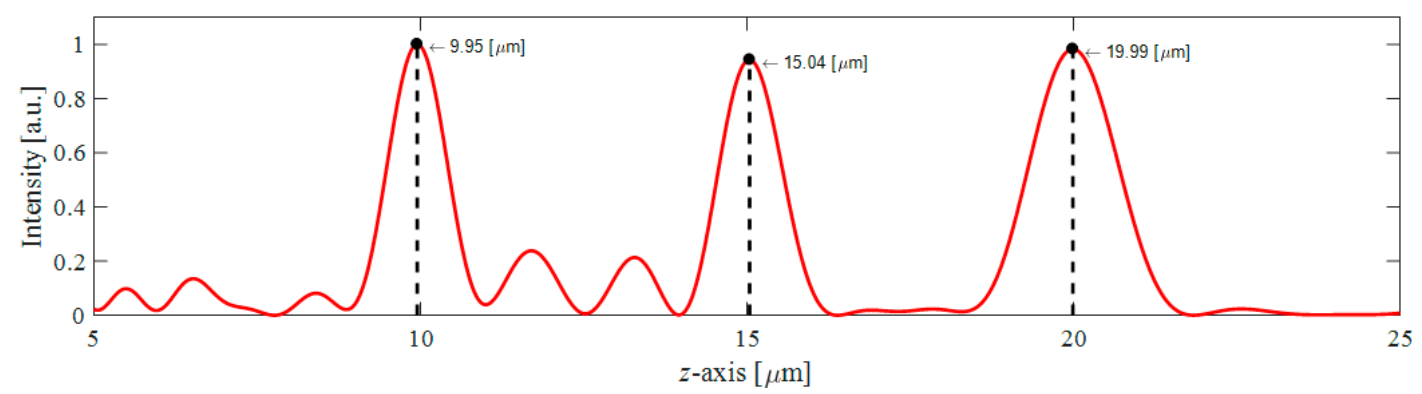

(c)

Figure 2. Simulation results on the monochromatic, multi-focal MFZP specified in Table 1: (a) Shape of the calculated MFZP pattern. (b) Calculated intensity pattern formed through the MFZP. (c) Calculated intensity profile on the center axis of the MFZP, i.e., the $z$-axis.

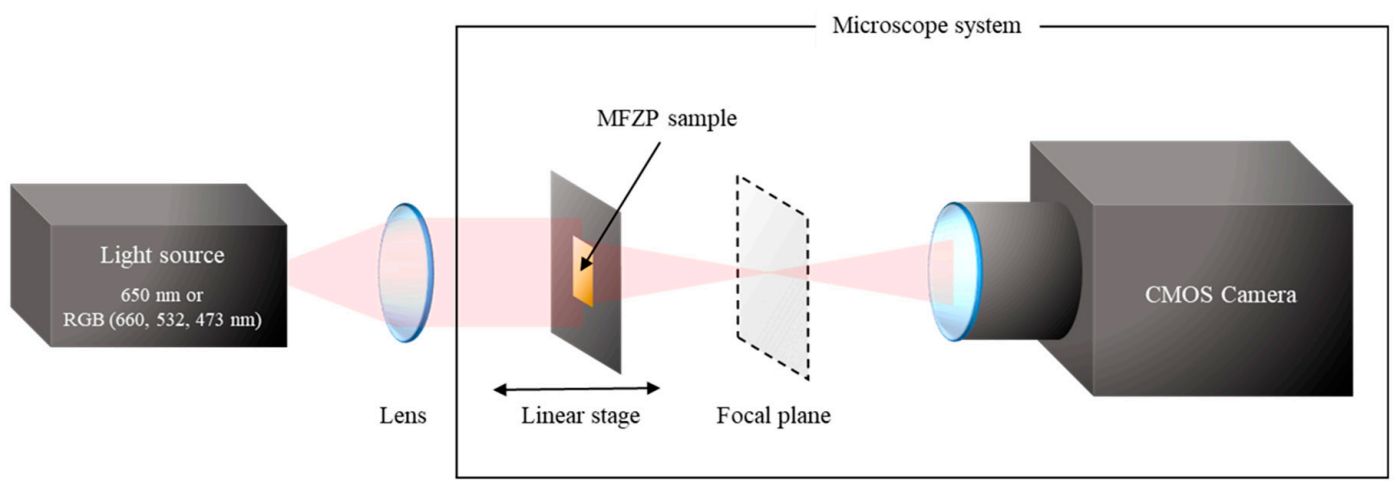

Figure 3. Schematic of the experimental arrangement for the characterization of MFZPs.

In Figure 4a,b, we illustrate the scanning electron microscope (SEM) image of the fabricated MFZP and the images of the transmitted light obtained at some specific distances by means of the optical microscope, respectively. One can see that the fabricated MFZP effectively produced multiple hot spots 
at the locations as closely as designed. In Figure $4 \mathrm{c}, \mathrm{d}$, we also illustrate the whole spatial distribution of the transmitted light in both the longitudinal and transverse directions, from which one can clearly see that the three hot spots were well separated and centered at the targeted locations. However, we could not resolve out even finer intensity distributions than detected owing to the limited sensitivity of the CMOS sensor array of the optical microscope system we used. We observed that the peak locations of the three hot spots took place exactly at 10,15, and $20 \mu \mathrm{m}$ with a measurement resolution uncertainty of $0.2 \mu \mathrm{m}$ and that the relative peak intensity levels of their average values were $0.97,0.96$, and 1.03 , respectively. We emphasize that, overall, the experimental results were in good agreement with the numerical results shown Figure 2c, indicating that the designed MFZP pattern was also completely feasible in terms of fabrication.

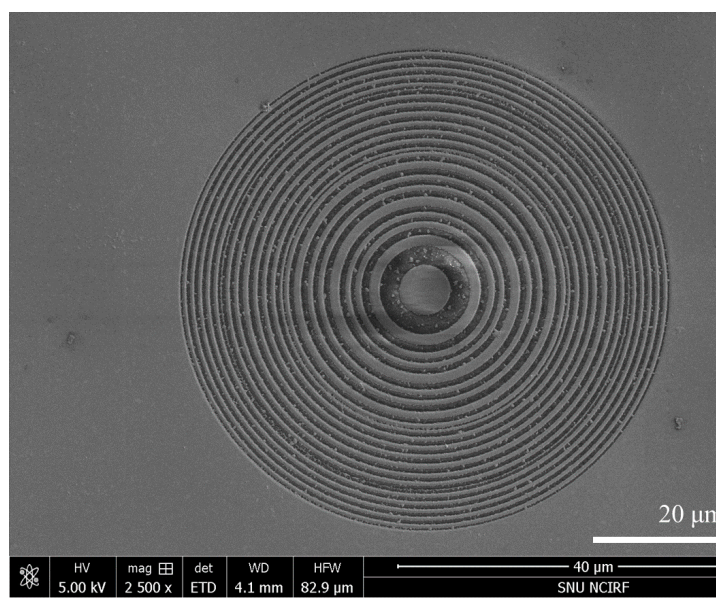

(a)

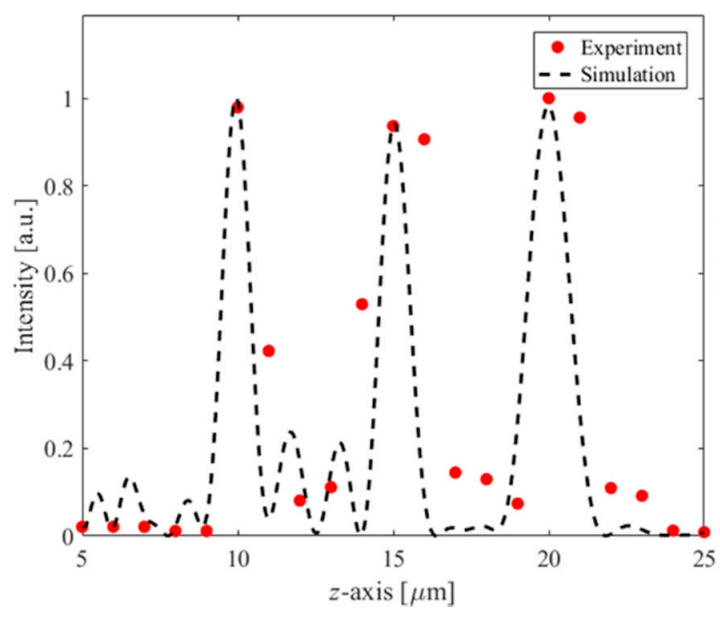

(c)

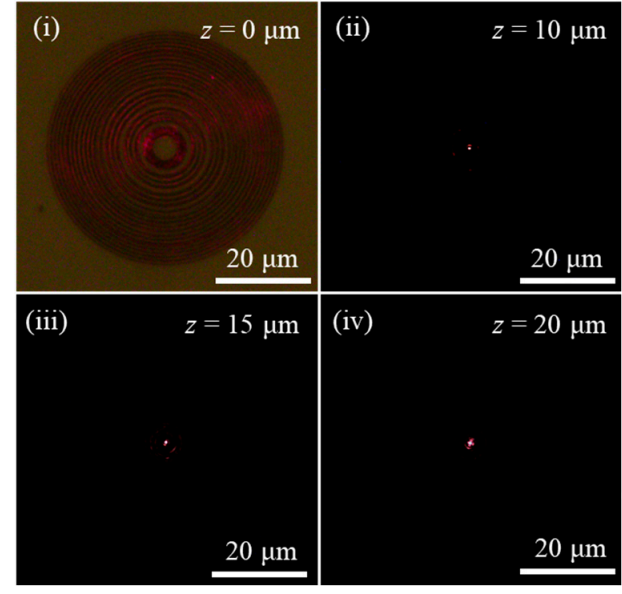

(b)

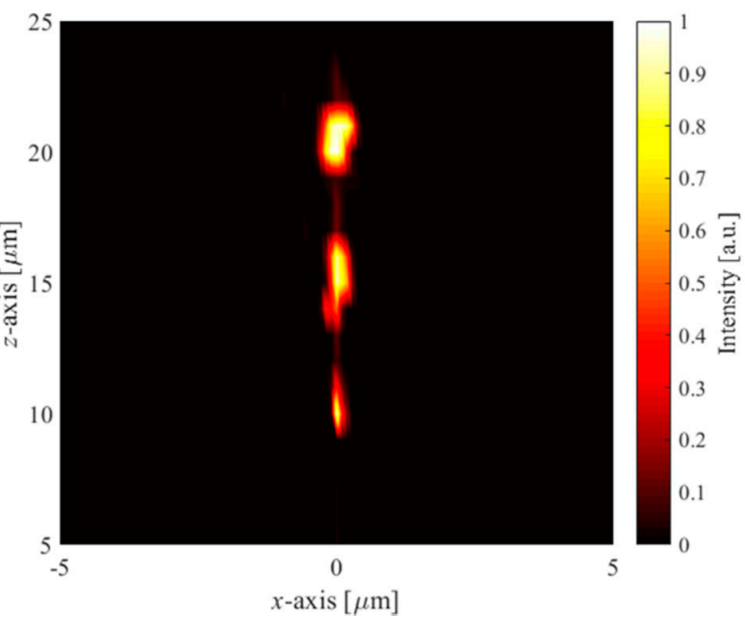

(d)

Figure 4. Experimental characterization of the fabricated monochromatic multi-focal MFZP: (a) SEM image of the fabricated MFZP. (b) Images of the transmitted light through the MFZP with respect to the longitudinal position taken by the optical microscope system for monochromatic incidence at $650 \mathrm{~nm}$ : (i) $z=0 \mu \mathrm{m}$, (ii) $z=10 \mu \mathrm{m}$, (iii) $z=15 \mu \mathrm{m}$, and (iv) $z=20 \mu \mathrm{m}$. (c) Comparison between the experimental and numerical results regarding the intensity profile on the center axis of the MFZP, i.e., the $z$-axis. (d) Whole spatial distribution of the transmitted light in both longitudinal and transverse directions.

\section{A Multi-Chromatic Mono-Focal MFZP}

It is worth noting that a conventional MFZP has a high chromatic aberration such that the effective focal length varies with a wavelength with a negative slope [32]. In other words, the longer 
the wavelength, the shorter the focal length. This feature can readily be seen from the relation between $f_{0}$ and $\lambda_{0}$ in Equation (1) and can also be verified in the simulation results shown in Figure 5, where we numerically analyzed the focusing characteristics of the monochromatic multi-focal MFZP designed for $650 \mathrm{~nm}$ in the preceding section, extending the operating wavelength range from 600 to $700 \mathrm{~nm}$. Although there were a number of side lobes of low intensity levels, we noted that there were three main traces with negative slopes that could lead to significant hot spots at three different locations for a fixed wavelength. Considering the negative slopes, one can also find three different wavelengths of incident light that can simultaneously lead to producing a hot spot at a fixed distance. Specifically, if one draws a horizontal line in Figure 5, it will intersect the three main traces at three different wavelengths. This means that a monochromatic multi-focal MFZP can in principle also function as a multi-chromatic mono-focal MFZP that simultaneously produces a hot spot at an identical location for multiple wavelengths, e.g., multi-color or RGB beams. In order to produce such a hot spot at a desired location for desired multiple wavelengths, we once again could exploit the PSR via the VPSs discussed in the preceding section. In this case, all we had to do was place all the VPSs at an identical location but with different wavelengths.

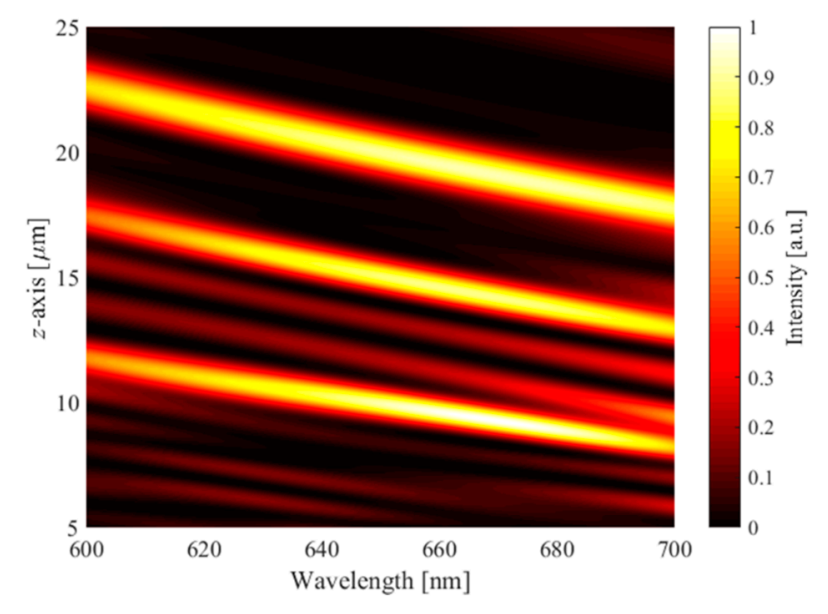

Figure 5. Numerical simulation of the monochromatic multi-focal MFZP specified in Table 1, regarding the normalized intensity distribution along the $z$-axis with respect to incident wavelength.

To demonstrate a proof of principle for multi-chromatic, mono-focal functionality, we designed and fabricated another MFZP capable of simultaneously producing a hot spot at an identical location for RGB beams. We assumed that the wavelengths of the RGB colors were 660, 532, and $473 \mathrm{~nm}$, respectively, and that the target focal point was located at $15 \mu \mathrm{m}$ away from the MFZP plane. In a similar manner to the design procedure taken in the preceding section, we carried out the application of the PSR via the VPSs along with the PSO algorithm. We note that in this case we took a single indicator for optimization only from the perspective of how similarly the three focal points were formed at the desired location in terms of $\Delta f_{m}$ with a relative tolerance value of $\pm 0.1 \%$. It is worth noting that we did not consider a second criterion on $\Delta I_{m}$ at this stage, mainly because the incident multi-color beams do not necessarily have the same power levels. We summarize all the simulation parameters in Table 2.

In Figure 6, we show simulation results in which one can see that all the RGB colors were focused on a nearly identical location at $15 \mu \mathrm{m}$. The minimum side lobe suppression ratio for all the cases was $-3.47 \mathrm{~dB}$, which was relatively higher than that of the monochromatic multi-focal MFZP. However, we note that one can improve it significantly if one extends the optimization procedure with an additional criterion. In addition, the relative peak intensity levels of blue and green colors to that of red were 0.73 and 0.76 , respectively, which was due to the fact that we did not put any indicator in the PSO procedure that took into account the relative peak intensity levels among colors. In order to adjust 
them further, one can take the recourse of optimization by putting additional tolerance on $\Delta I_{m}$, as was done in the preceding section.

Table 2. Simulation parameters for a multi-chromatic, mono-focal MFZP.

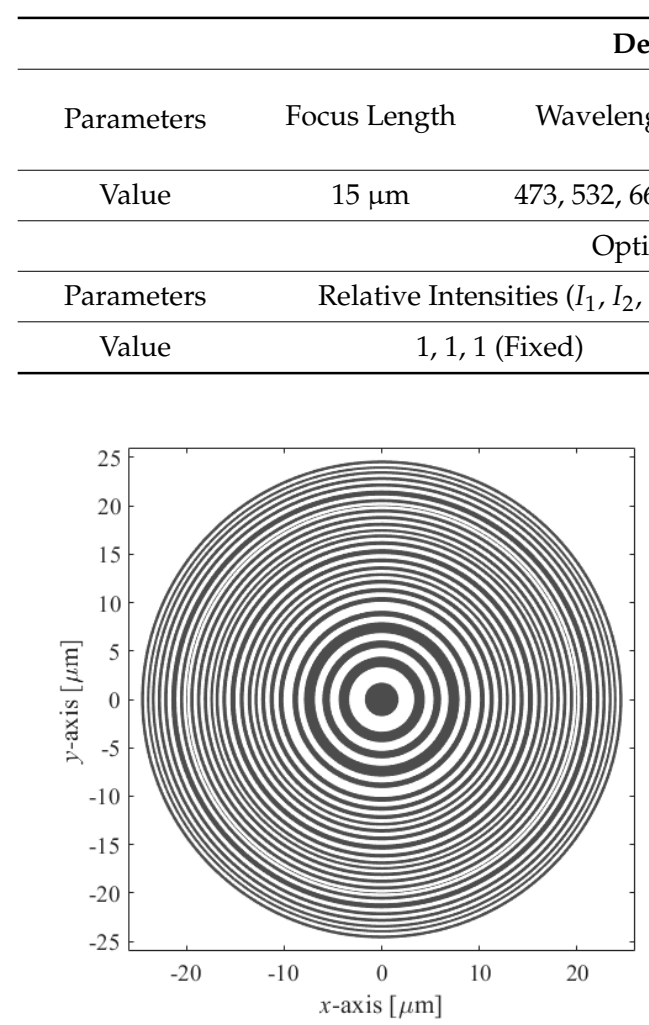

(a)
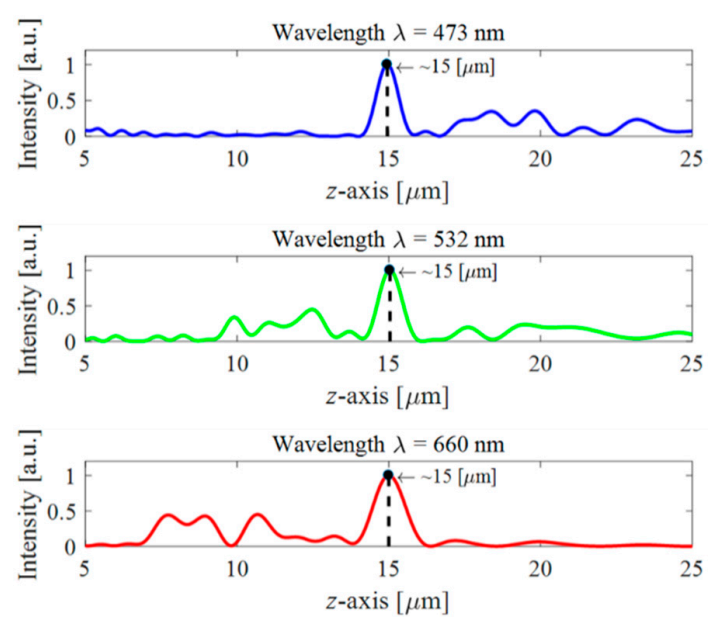

(b)
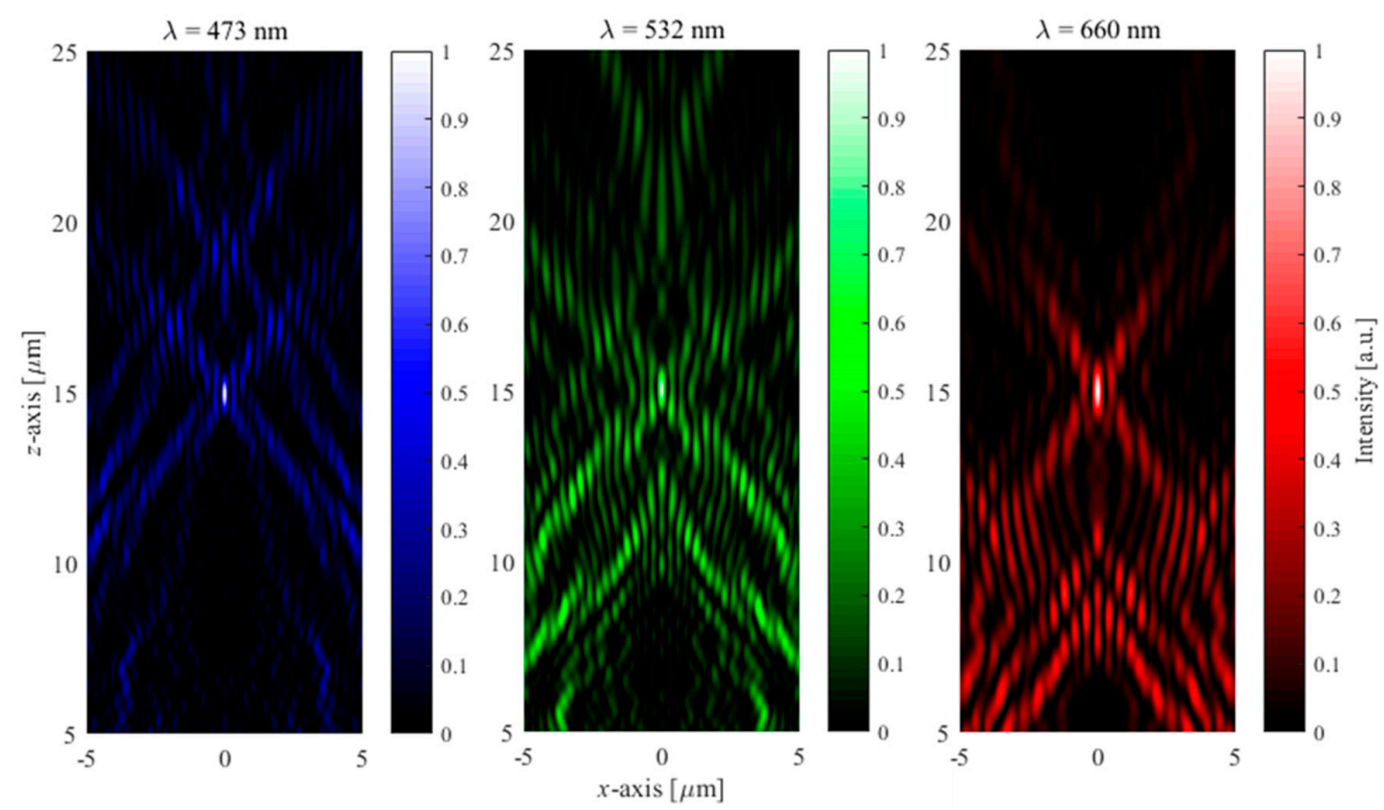

(c)

Figure 6. Simulation results for the multi-chromatic mono-focal MFZP specified in Table 2: (a) Shape of the calculated MFZP pattern. (b) Calculated intensity profiles on the center axis of the MFZP, i.e., the $z$-axis, for RGB beams. (c) Calculated intensity pattern formed through the MFZP for RGB beams. 
Based on the designed pattern shown in Figure 6a, we fabricated an MFZP with multi-chromatic mono-focal functionality, for which we used the electron beam evaporation and the FIB milling processes, as discussed in the preceding section. We characterized it experimentally, using the same setup as illustrated in Figure 3 in which we replaced the $650 \mathrm{~nm}$ laser diode with an RGB laser system. The RGB laser system was constructed by combining individual red (Flamenco 300, Cobolt, Solna, Sweden), green (Verdi G2 SLM, Coherent, Palo Alto, CA, USA), and blue (Excelsior 473, Spectra-Physics, Santa Clara, CA, USA) laser beams operating at 660, 532, and $473 \mathrm{~nm}$, respectively, using dichroic mirrors.

In Figure 7a,b, we illustrate the SEM image of the fabricated MFZP and the images of the hot spots obtained using the optical microscope for individual color beams as well as for the combined RGB beams at the same location, i.e., $z=\sim 15 \mu \mathrm{m}$, respectively. One can see that the fabricated MFZP effectively produced hot spots at the same location for all the RGB beams. In particular, when the individual RGB beams were illuminated on the MFZP at the same time, one can clearly see a single bright hot spot in white color at the center, which means that the three hot spots in RGB colors were formed at the same location. Owing to the limited sensitivity of the CMOS sensor array of the optical microscope system we used, we could not resolve out even finer intensity distributions than detected, except for some low intensity side lobes in the transverse plane. Notwithstanding this, we emphasize that overall the experimental results were in good agreement with the numerical results presented in Figure 6.

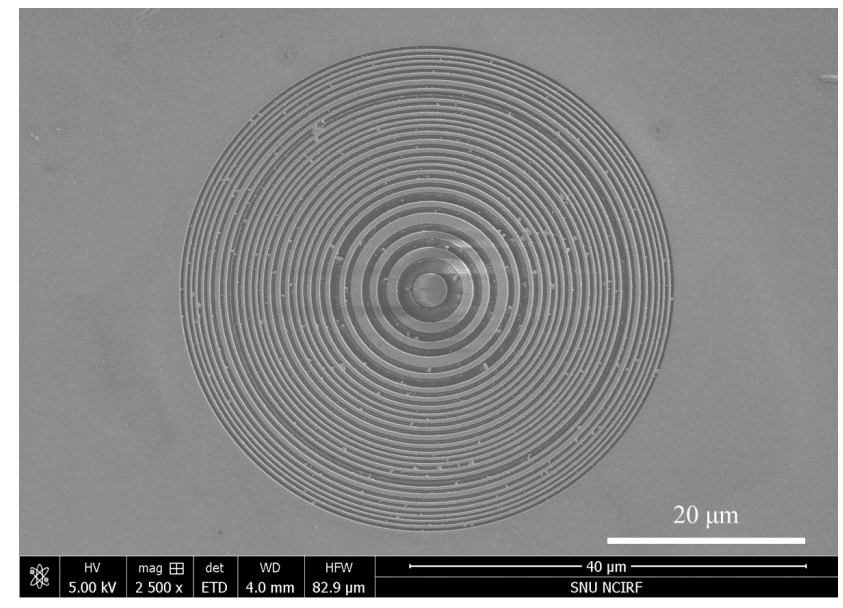

(a)

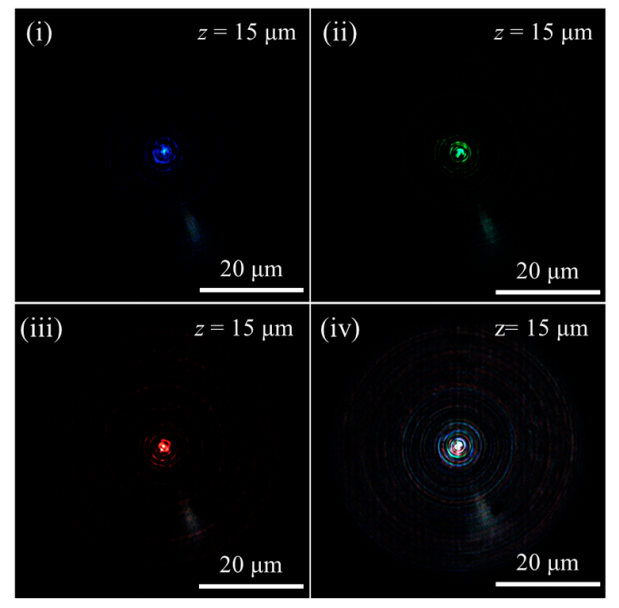

(b)

Figure 7. Experimental characterization of the fabricated multi-chromatic mono-focal MFZP: (a) SEM image of the fabricated MFZP. (b) Images of the transmitted light through the MFZP at $z=15 \mu \mathrm{m}$ with respect to incidence wavelength: (i) $\lambda=473 \mathrm{~nm}$ (blue), (ii) $\lambda=532 \mathrm{~nm}$ (green), (iii) $\lambda=660 \mathrm{~nm}$ (red), and (iv) combined RGB incidence (white).

\section{Conclusions}

We have demonstrated a monochromatic, multi-focal MFZP and a multi-chromatic, mono-focal MFZP using a novel design principle. This novel design principle is based on the PSR by implementing VPSs at the desired focal points. We utilized the PSO algorithm to determine the appropriate intensity levels and phase offset values for individual VPSs, thereby optimizing the resultant MFZP pattern from the perspective of both multi-focal functionality and fabrication feasibility. Based on the proposed design principle, we first devised a monochromatic multi-focal MFZP for $650 \mathrm{~nm}$ incident light, capable of producing multiple hotspots at 10,15, and $20 \mu \mathrm{m}$ with a relative tolerance of the focal distance of $<0.5 \%$ and with a relative peak intensity tolerance of $<2.9 \%$. We experimentally implemented the designed MFZP pattern onto an Au-coated glass substrate, utilizing electron beam evaporation deposition and the FIB milling processes. We characterized it with a $650 \mathrm{~nm}$ laser source and an imaging 
apparatus based on an optical microscope with a translation stage. We verified that the measured effective focal distances and relative peak intensity levels for individual hot spots were in good agreement with the numerically calculated results. We then extended the proposed design principle to the case of a multi-chromatic, mono-focal MFZP, which was capable of simultaneously producing a hot spot at an identical location for RGB wavelengths of 660, 532, and $473 \mathrm{~nm}$. We numerically verified that it could produce a single hot spot at a focal distance of $15 \mu \mathrm{m}$ for the three RGB wavelengths with relative tolerance of the focal distance of $<0.07 \%$. We fabricated and experimentally characterized it in a similar manner. We verified that the measured multi-chromatic, mono-focal characteristics were also in good agreement with the numerical results.

Both the monochromatic, multi-focal MFZP and the multi-chromatic, mono-focal MFZP can have various applications, such as laser micro-machining, optical trapping, biomedical sensing, confocal collimation, achromatic optics, etc. All of these invariably require novel characteristics in terms of beam focusing. Moreover, the proposed design principle based on the PSR via VPSs should be a very effective way of systematically designing various MFZP devices with novel functionality. In addition, although we only utilized the PSO algorithm for the optimization of the intensity and phase offset parameters of the VPSs, various other algorithms, such as deep learning techniques, can also be exploited in order to improve optimization performance and efficiency.

Acknowledgments: This work was supported in part by a National Research Foundation of Korea (NRF) grant funded by the government of Korea (2017R1D1A1B03036201); the Ministry of Trade, Industry and Energy (Project no. 10060150); and the Brain Korea 21 Plus Program.

Author Contributions: Jinseob Kim performed the experiments and numerical simulations and wrote the manuscript. Hyuntai Kim contributed to the experiments and fabrications. Gun-Yeal Lee prepared the samples and supported the experiments. Juhwan Kim contributed to the numerical simulations. Byoungho Lee supervised Gun-Yeal Lee's work. Yoonchan Jeong led and supervised Jinseob Kim, Hyuntai Kim and Juhwan Kim's work and revised the manuscript.

Conflicts of Interest: The authors declare no conflict of interest.

\section{References}

1. Young, M. Zone plates and their aberrations. J. Opt. Soc. Am. 1972, 62, 972-976. [CrossRef]

2. Xu, Y.; Lu, Y.; Zhu, Z.; Shi, J.; Guan, C.; Yuan, L. Multimode fiber focusing lens based on plasmonic structures. In Proceedings of the Optoelectronic Devices and Integration VI, Beijing, China, 12-14 October 2016; Volume 10019, p. 100191C.

3. Wan, W.; Ma, C.; Liu, Z. Control the dispersive properties of compound plasmonic lenses. Opt. Commun. 2013, 291, 390-394. [CrossRef]

4. Jia, J.; Xie, C.; Liu, M.; Wan, L. A super-resolution Fresnel zone plate and photon sieve. Opt. Laser Eng. 2010, 48, 760-765. [CrossRef]

5. Kim, H.; An, H.; Kim, J.; Lee, S.; Park, K.; Lee, S.; Hong, S.; Vazquez-Zuniga, L.A.; Lee, S.Y.; Lee, B.; et al. Corrugation-assisted metal-coated angled fiber facet for wavelength-dependent off-axis directional beaming. Opt. Express 2017, 25, 8366-8385. [CrossRef] [PubMed]

6. Kim, H.; Lee, S.Y.; Koo, S.; Kim, J.; Park, K.; Lee, D.; Vazquez-Zuniga, L.A.; Park, N.; Lee, B.; Jeong, Y. Theoretical study on the generation of a low-noise plasmonic hotspot by means of a trench-assisted circular nano-slit. Opt. Express 2014, 22, 26844-26853. [CrossRef] [PubMed]

7. Li, M.; Li, W.; Li, H.; Zhu, Y.; Yu, Y. Controllable design of super-oscillatory lenses with multiple sub-diffraction-limit foci. Sci. Rep. 2017, 7, 1335. [CrossRef] [PubMed]

8. Yuan, G.H.; Rogers, E.T.; Zheludev, N.I. Achromatic super-oscillatory lenses with sub-wavelength focusing. Light Sci. Appl. 2017, 6, e17036. [CrossRef]

9. Rogers, E.T.; Savo, S.; Lindberg, J.; Roy, T.; Dennis, M.R.; Zheludev, N.I. Super-oscillatory optical needle. Appl. Phys. Lett. 2013, 102, 031108. [CrossRef]

10. Wróbel, P.; Pniewski, J.; Antosiewicz, T.J.; Szoplik, T. Focusing radially polarized light by a concentrically corrugated silver film without a hole. Phys. Rev. Lett. 2009, 102, 183902. [CrossRef] [PubMed] 
11. Monsoriu, J.A.; Calatayud, A.; Remón, L.; Furlan, W.D.; Saavedra, G.; Andrés, P. Bifocal Fibonacci diffractive lenses. IEEE Photonics J. 2013, 5, 3400106. [CrossRef]

12. Saavedra, G.; Furlan, W.D.; Monsoriu, J.A. Fractal zone plates. Opt. Lett. 2003, 28, 971-973. [CrossRef] [PubMed]

13. Ferrando, V.; Calatayud, A.; Giménez, F.; Furlan, W.D.; Monsoriu, J.A. Cantor dust zone plates. Opt. Express 2013, 21, 2701-2706. [CrossRef] [PubMed]

14. Venkatakrishnan, K.; Tan, B. Interconnect microvia drilling with a radially polarized laser beam. J. Micromech. Microeng. 2006, 16, 2603-2607. [CrossRef]

15. Neuman, K.C.; Block, S.M. Optical trapping. Rev. Sci. Instrum. 2004, 75, 2787-2809. [CrossRef] [PubMed]

16. Liu, Z.; Wei, Y.; Zhang, Y.; Sun, B.; Zhao, E.; Zhang, Y.; Yang, J.; Yuan, L. A novel surface plasmon resonance sensor based on fiber butt-joint technology. Sens. Actuator B Chem. 2015, 221, 1330-1334. [CrossRef]

17. Kim, H.; Vazquez-Zuniga, L.A.; Kim, J.; Park, K.; Lee, D.; Hong, S.; Jeong, Y. Fiberized plasmonic Fresnel zone plate for wavelength dependent position tunable optical trapping. In Proceedings of the 11th Conference on Lasers and Electro-Optics Pacific Rim, Busan, Korea, 24-28 August 2015; p. 28E2_1.

18. Ribeiro, R.S.R.; Dahal, P.; Guerreiro, A.; Jorge, P.A.; Viegas, J. Fabrication of Fresnel plates on optical fibres by FIB milling for optical trapping, manipulation and detection of single cells. Sci. Rep. 2017, 7, 4485.

19. Moh, K.J.; Yuan, X.C.; Bu, J.; Zhu, S.W.; Gao, B.Z. Radial polarization induced surface plasmon virtual probe for two-photon fluorescence microscopy. Opt. Lett. 2009, 34, 971-973. [CrossRef] [PubMed]

20. Jiang, T.; Chen, Q.D.; Zhang, J.; Tian, Z.N.; Niu, L.G.; Li, Q.S.; Wang, H.Y.; Qin, L.; Sun, H.B. Monolithic bifocal zone-plate lenses for confocal collimation of laser diodes. Opt. Lett. 2013, 38, 3739-3742. [CrossRef] [PubMed]

21. Wang, Y.; Yun, W.; Jacobsen, C. Achromatic Fresnel optics for wideband extreme-ultraviolet and X-ray imaging. Nature 2003, 424, 50-53. [CrossRef] [PubMed]

22. Simpson, M.J.; Michette, A.G. Imaging properties of modified Fresnel zone plates. Opt. Acta Int. J. Opt. 1984, 31, 403-413. [CrossRef]

23. Gao, N.; Xie, C.; Li, C.; Jin, C.; Liu, M. Square optical vortices generated by binary spiral zone plates. Appl. Phys. Lett. 2011, 98, 151106. [CrossRef]

24. Tao, S.H.; Yuan, X.C.; Lin, J.; Burge, R.E. Sequence of focused optical vortices generated by a spiral fractal zone plate. Appl. Phys. Lett. 2006, 89, 031105. [CrossRef]

25. Huang, J.; Murai, Y.; Yamamoto, F. Shallow DOF-based particle tracking velocimetry applied to horizontal bubbly wall turbulence. Flow Meas. Instrum. 2008, 19, 93-105. [CrossRef]

26. Khorasaninejad, M.; Shi, Z.; Zhu, A.Y.; Chen, W.T.; Sanjeev, V.; Zaidi, A.; Capasso, F. Achromatic metalens over $60 \mathrm{~nm}$ bandwidth in the visible and metalens with reverse chromatic dispersion. Nano Lett. 2017, 17, 1819-1824. [CrossRef] [PubMed]

27. Saleh, B.E.; Teich, M.C.; Saleh, B.E. Fundamentals of Photonics, 2nd ed.; Wiley: New York, NY, USA, 2007; p. 110.

28. Lerosey, G.; De Rosny, J.; Tourin, A.; Derode, A.; Montaldo, G.; Fink, M. Time reversal of electromagnetic waves. Phys. Rev. Lett. 2004, 92, 193904. [CrossRef] [PubMed]

29. Kennedy, J.; Eberhart, R. Particle Swarm Optimization. In Proceedings of the IEEE International Conference on Neural Networks, Perth, Australia, 27 November-1 December 1995; pp. 1942-1948.

30. Harsha, K.S. Principles of Vapor Deposition of Thin Films, 1st ed.; Elsevier: Oxford, UK, 2006; p. 400.

31. Vesseur, E.J.R.; De Waele, R.; Lezec, H.J.; Atwater, H.A.; García de Abajo, F.J.; Polman, A. Surface plasmon polariton modes in a single-crystal Au nanoresonator fabricated using focused-ion-beam milling. Appl. Phys. Lett. 2008, 92, 083110. [CrossRef]

32. Kim, H.; Kim, J.; An, H.; Lee, Y.; Lee, G.Y.; Na, J.; Park, K.; Lee, S.; Lee, S.Y.; Lee, B.; et al. Metallic Fresnel zone plate implemented on an optical fiber facet for super-variable focusing of light. Opt. Express 2017, 25, 30290-30303. [CrossRef] [PubMed]

(C) 2018 by the authors. Licensee MDPI, Basel, Switzerland. This article is an open access article distributed under the terms and conditions of the Creative Commons Attribution (CC BY) license (http:/ / creativecommons.org/licenses/by/4.0/). 